\title{
Combined detection of serum UL16-binding protein 2 and macrophage inhibitory cytokine-1 improves early diagnosis and prognostic prediction of pancreatic cancer
}

\author{
YU-FEN ZHOU ${ }^{1}$, LING-XIAO XU ${ }^{1}$, LI-YA HUANG ${ }^{2}$, FANG GUO ${ }^{1}$, FAN ZHANG ${ }^{1}$, \\ XIANG-YI HE ${ }^{1}$, YAO-ZONG YUAN ${ }^{1}$ and WEI-YAN YAO ${ }^{1}$ \\ ${ }^{1}$ Department of Gastroenterology, Ruijin Hospital Affiliated to Shanghai Jiaotong University School of Medicine, Shanghai; \\ ${ }^{2}$ Department of Gastroenterology, General Hospital of Ningxia Medical University, Yinchuan, Ningxia, P.R. China
}

Received December 3, 2013; Accepted July 8, 2014

DOI: $10.3892 / \mathrm{ol} .2014 .2429$

\begin{abstract}
Pancreatic cancer (PC) is the fourth leading cause of cancer-related mortality in the United States. There is no effective serum biomarker for the early diagnosis of PC at present. Although serum UL16-binding protein 2 (ULBP2) and macrophage inhibitory cytokine-1 (MIC-1) levels are reported to be elevated in $\mathrm{PC}$ patients, the diagnostic and prognostic value of ULBP2 and MIC-1 alone or in combination remains unknown. The aim of the present case-control study was to compare the diagnostic value of ULBP2, MIC-1 and carbohydrate antigen 19-9 (CA19-9) in 359 serum samples, consisting of 152 cases of PC, 20 cases of pre-pancreatic cancer, 91 cases of chronic pancreatitis (CP) and 96 normal controls (NC). All patients were followed up for a median of 2 years. It was found that the serum levels of ULBP2, MIC-1 and CA19-9 were significantly higher in the PC patients compared with those in the NC group. In distinguishing PC from the $\mathrm{CP}$, the highest sensitivity and specificity were ULBP2 (0.878) and CA19-9 (0.816), respectively. The area under the receiver operating characteristic curve of ULBP2 was 0.923 , which was the highest of the three biomarkers. MIC-1 was the optimal choice for the diagnosis of early-stage PC (area under the curve, 0.831). Overall, MIC-1 in combination with ULBP2 improved the diagnostic accuracy in differentiating $\mathrm{PC}$ from $\mathrm{CP}$ and $\mathrm{NC}$. In addition, a higher level of MIC-1 was correlated with a poorer prognosis, as calculated by the Kaplan-Meier test $(\mathrm{P}=0.039)$. Patients with serum MIC-1 levels of $\geq 1,932 \mathrm{ng} / \mathrm{ml}$ had a median survival time of $15.62 \pm 2.44$ months (mean \pm standard deviation) vs. $18.66 \pm 2.43$ months in patients with a lower level
\end{abstract}

Correspondence to: Dr Wei-Yan Yao, Department of Gastroenterology, Ruijin Hospital Affiliated to Shanghai Jiaotong University School of Medicine, 197 Ruijin Er Road, Shanghai 200025, P.R. China

E-mail: yaowymail@sina.com

Key words: macrophage inhibitory cytokine-1, serum biomarker, pancreatic cancer, UL16-binding protein 2 of MIC-1. Overall, combined detection of serum MIC-1 and ULBP2 improved the diagnostic accuracy in differentiating PC from CP and NC, and serum MIC-1 level alone was a predictor of survival in the patients with $\mathrm{PC}$.

\section{Introduction}

Pancreatic cancer (PC) is an aggressive malignancy with an overall five-year survival rate of only $\sim 5 \%$ (1). The morbidity of PC is ranked tenth while mortality is ranked fourth among all cancers (2). The majority of PC patients have already lost the opportunity for radical resection at the time of diagnosis (1). The median survival time of patients with advanced $\mathrm{PC}$ is less than six months, while the five-year survival rate of resected minute pancreatic cancers $(\leq 10 \mathrm{~mm})$ can be $>75 \%$ (3). Therefore, the early diagnosis of PC is of great significance to improve patient outcome.

Yachida et al (4) recorded a period of at least 15 years between the first mutation and the birth of the metastatic ability of PC. In addition, a retrospective study found that CT scans could diagnose asymptomatic PC six months prior to clinical diagnosis (5). However, no reliable non-invasive test is available at present for the early diagnosis of PC.

UL16-binding protein 2 (ULBP2) belongs to the ULBP family. The ULBPs are ligands for natural killer group 2, member D (NKG2D)/DNAX-activating protein of $10 \mathrm{kDa}$, an activating receptor expressed by natural killer (NK) cells (6). Macrophage inhibitory cytokine-1 (MIC-1) is a novel member of the TGF- $\beta$ family (7). Knowing that ULBP2 and MIC-1 are highly expressed in $\mathrm{PC}$, the aim of the present case-control study was to evaluate the diagnostic and prognostic value of ULBP2, MIC-1 and CA19-9 in PC.

\section{Material and methods}

Study design. A total of 359 subjects were enrolled in this study, consisting of 152 cases of PC, 20 cases of pre-pancreatic cancer (PPC; pancreatic intraepithelial neoplasia, intraductal papillary mucinous neoplasm and mucinous cystic neoplasm), 91 cases of chronic pancreatitis (CP) and 96 normal controls (NC). All the patients were admitted between 2009 and 2012 to 
Table I. Demographics and clinicopathologic characteristics of the patients.

\begin{tabular}{|c|c|c|c|c|}
\hline Characteristic & $\mathrm{PC}$ & $\mathrm{CP}$ & $\mathrm{NC}$ & PPC \\
\hline No. of patients & 152 & 91 & 96 & 20 \\
\hline \multicolumn{5}{|l|}{ Age } \\
\hline Mean (SD), years & $56(13.5)$ & $58(15.0)$ & $58(7.6)$ & $60(11.3)$ \\
\hline$>60, \mathrm{n}$ & 88 & 58 & 53 & 6 \\
\hline$\leq 60, \mathrm{n}$ & 64 & 33 & 43 & 14 \\
\hline \multicolumn{5}{|l|}{ Gender, $\mathrm{n}$} \\
\hline Males & 101 & 52 & 72 & 15 \\
\hline Females & 51 & 39 & 24 & 5 \\
\hline \multicolumn{5}{|l|}{ Stage, $\mathrm{n}$} \\
\hline IA & 5 & & & \\
\hline IB & 12 & & & \\
\hline IIA & 36 & & & \\
\hline IIB & 20 & & & \\
\hline III & 40 & & & \\
\hline IV & 39 & & & \\
\hline CA19-9, U/ml ${ }^{\mathrm{a}}$ & $1448.78 \pm 3707.04$ & $38.23 \pm 138.96$ & $7.69 \pm 4.89$ & $10.44 \pm 7.40$ \\
\hline $\mathrm{CEA}, \mathrm{ng} / \mathrm{ml}^{\mathrm{a}}$ & $15.15 \pm 94.79$ & $2.74 \pm 2.09$ & $1.27 \pm 2.05$ & $2.63 \pm 1.25$ \\
\hline
\end{tabular}

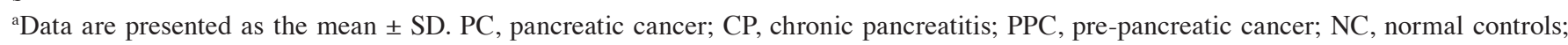
CA19-9, carbohydrate antigen 19-9; CEA, carcinoembryonic antigen; SD, standard deviation.

the Department of Gastroenterology, Ruijin Hospital Affiliated to Shanghai Jiaotong University School of Medicine (Shanghai, China). The end-point of the follow-up period was April 2013, and a total of 100 follow-up records from the 152 PC patients were obtained. The study was approved by The Ethics Committee of Ruijin Hospital Affiliated to Shanghai Jiaotong University School of Medicine and all enrolled subjects signed informed consent documents.

The cytological or pathological evidence from the PC patients was obtained by endoscopic ultrasound, endoscopic retrograde cholangiopancreatography or surgery. PPC was diagnosed by pathology (8). Serum samples of the PC and PPC groups were collected prior to treatment. According to the sixth edition of the standard proposed in 2002 by the American Joint Committee on Cancer (9), tumor-node-metastasis (TNM) staging was performed on PC patients based on the post-operative pathological and CT scan findings. $\mathrm{CP}$ was defined based on clinical manifestations, including abdominal pain and steatorrhea, CT scan findings of calcifications, irregular pancreatograms and CP pathological changes obtained by surgery or secretin stimulation test to exclude PC. Baseline demographic information for all groups is detailed in Table I.

Measurement of ULBP2 and MIC-1. All serum samples were frozen at $-80^{\circ} \mathrm{C}$ until use. The serum levels of ULBP2 and MIC-1 were measured quantitatively by sandwich ELISA according to the manufacturer's instructions, using the DuoSet ELISA kit (R\&D Systems, Minneapolis, MN, USA) for human ULBP2 and MIC-1, respectively. The standard curve was plotted according to the manufacturer's instructions. The detectable range of ULBP2 was between $15.6 \mathrm{pg} / \mathrm{ml}$ and $1 \mathrm{ng} / \mathrm{ml}$, and the detectable range of MIC-1 was between $62.5 \mathrm{pg} / \mathrm{ml}$ and $4 \mathrm{ng} / \mathrm{ml}$. Each sample was placed into two wells and detected twice. If the result was beyond the detection range, the serum sample was re-determined following appropriate dilution. A microplate reader was used to analyze the result at $450 \mathrm{~nm}$, and corrected at $570 \mathrm{~nm}$. CA19-9 was determined according to the manufacturer's instructions for the solid phase radioimmunoassay in the Laboratory of Biochemical Medicine in the Ruijin Hospital Affiliated to Shanghai Jiaotong School of Medicine.

Statistical analysis. Data were analyzed using IBM SPSS statistic 19 (IBM, Armonk, NY, USA). The Wilcoxon rank sum test and bilateral test were used in pairwise comparison of CA19-9, ULBP2 and MIC-1 serum levels between the PC, $\mathrm{PPC}, \mathrm{CP}$ and $\mathrm{NC}$ groups. The correlation between the serum ULBP2 and MIC-1 levels and the patient characteristics in the PC group was evaluated by Spearman's correlation analysis. A logistic regression analysis was used to draw a receiver operating characteristic (ROC) curve. The area under the curve (AUC) was calculated to compare the performance of different serum biomarkers as a diagnostic test. Survival data were analyzed using the Kaplan-Meier method with a log-rank test for comparison of survival curves. $\mathrm{P}<0.05$ was considered to indicate a statistically significant difference.

\section{Results}

Serum ULBP2, MIC- and CA19-9 levels in each group. The serum ULBP2, MIC-1 and CA19-9 levels, shown for each group 
Table II. Comparison of serum ULBP2, MIC-1 and CA19-9 within groups, mean \pm standard deviation.

\begin{tabular}{lcccc}
\hline Biomarkers & PC $(\mathrm{n}=152)$ & $\mathrm{CP}(\mathrm{n}=91)$ & $\mathrm{PPC}(\mathrm{n}=20)$ & $\mathrm{NC}(\mathrm{n}=96)$ \\
\hline $\mathrm{CA} 19-9, \mathrm{U} / \mathrm{ml}$ & $1448.78 \pm 3707.04$ & $38.23 \pm 138.96$ & $10.44 \pm 7.40$ & $7.69 \pm 4.89$ \\
$\mathrm{ULBP} 2, \mathrm{pg} / \mathrm{ml}$ & $219.89 \pm 182.48$ & $68.33 \pm 36.78$ & $76.51 \pm 40.9$ & $62.62 \pm 11.37$ \\
$\mathrm{MIC}-1, \mathrm{pg} / \mathrm{ml}$ & $3521.34 \pm 3903.38$ & $959.61 \pm 878.98$ & $973.59 \pm 588.89$ & $427.61 \pm 316.95$
\end{tabular}

PC, pancreatic cancer; CP, chronic pancreatitis; PPC, pre-pancreatic cancer; NC, normal controls; CA19-9, carbohydrate antigen 19-9; ULBP2, UL16-binding protein 2; MIC-1, macrophage inhibitory cytokine-1.

Table III. Sensitivity, specificity and AUC of different biomarkers in distinguishing between PC, CP and NC.

\begin{tabular}{|c|c|c|c|c|c|}
\hline Markers (optimal cut-off) & AUC & $95 \% \mathrm{CI}$ & P-value & Sensitivity & Specificity \\
\hline \multicolumn{6}{|l|}{ PC vs. CP } \\
\hline MIC-1 (983.20 pg/ml) & 0.820 & $0.753-0.887$ & $<0.001$ & 0.806 & 0.632 \\
\hline ULBP2 (86.12 pg/ml) & 0.923 & $0.871-0.975$ & $<0.001$ & 0.878 & 0.816 \\
\hline CA19-9 (36.2 U/ml) & 0.799 & $0.728-0.870$ & $<0.001$ & 0.763 & 0.816 \\
\hline \multicolumn{6}{|l|}{$\mathrm{PC}$ vs. NC } \\
\hline MIC-1 (642.83 pg/ml) & 0.958 & 0.924-0.992 & $<0.001$ & 0.899 & 0.903 \\
\hline ULBP2 (94.08 pg/ml) & 0.889 & $0.824-0.955$ & $<0.001$ & 0.820 & 0.806 \\
\hline CA19-9 (18.44 U/ml) & 0.883 & $0.833-0.932$ & $<0.001$ & 0.820 & 0.968 \\
\hline
\end{tabular}

PC, pancreatic cancer; CP, chronic pancreatitis; PPC, pre-pancreatic cancer; NC, normal controls; CA19-9, carbohydrate antigen 19-9; ULBP2, UL16-binding protein 2; MIC-1, macrophage inhibitory cytokine-1; AUC, area under the curve; CI, confidence interval.

in Table II, were significantly higher in the PC patients than in the $\mathrm{NC}$ group $(\mathrm{P}<0.0001)$. In addition, there was a significant difference in the serum ULBP2 and MIC-1 levels between the $\mathrm{PPC}$ and $\mathrm{NC}$ groups $(\mathrm{P}=0.001$ and $\mathrm{P}=0.003$, respectively), but there was no statistically significant difference in serum CA19-9 between the two groups $(\mathrm{P}=0.063)$. There was no significant correlation between the ULBP2 levels and age, gender, tumor location, $\mathrm{T}$ stage, $\mathrm{N}$ stage, $\mathrm{M}$ stage or TNM stage in the PC group, although serum MIC-1 was significantly correlated with $\mathrm{T}$ stage $(\mathrm{P}=0.014)$.

Comparison of diagnostic performances of ULBP2, MIC-1 and CA19-9 alone or a combination of two or three of these markers for PC diagnosis. Subsequently, the diagnostic performance of ULBP2, MIC-1 and CA19-9 alone was evaluated by plotting ROC curves and calculating the AUC. The optimal cut-off point was determined by ROC curve analysis. The sensitivity, specificity and AUC of different biomarkers in distinguishing PC from CP or NC are shown in Table III. The P-value of the AUC of the different groups was $<0.001$ in all groups. ULBP2 performed the best in the differential diagnosis of PC and CP, and also had the best sensitivity and specificity given the cut-off of ULBP2 at $86.12 \mathrm{pg} / \mathrm{ml}$ (Fig. 1A; Table III). Serum MIC-1 was effective in comparing PC and NC. When the cut-off values of CA19-9, ULBP2 and MIC-1 were set at $18.44 \mathrm{U} / \mathrm{ml}, 94.08 \mathrm{pg} / \mathrm{ml}$ and $642.83 \mathrm{pg} / \mathrm{ml}$ respectively, MIC-1 was found to have the highest sensitivity $(89.9 \%)$, while CA19-9 had the highest specificity $(96.8 \%)$ in distinguishing PC patients from NC (Fig. 1B; Table III).
Notably, MIC-1 was found to be the most effective marker [AUC, 0.964; 95\% confidence interval (CI), 0.929-0.999; $\mathrm{P}<0.001]$ in distinguishing between stage 1-2 PC and $\mathrm{NC}$. MIC-1 was also the most effective marker (AUC, 0.954; 95\% CI, 0.916-0.992; $\mathrm{P}<0.001)$ in distinguishing between stage 3-4 PC patients and NC. ULBP2 was the most effective marker (AUC, 0.925; 95\% CI, 0.873-0.978; $\mathrm{P}<0.001$ ) in distinguishing between stage 1-2 PC and CP patients. ULBP2 was also the most effective marker in distinguishing between stage 3-4 PC and CP patients (Fig. 1C-F; Table IV). Only MIC-1 had diagnostic capability in distinguishing between PPC and NC (AUC, 0.781; 95\% CI, 0.599-0.963; P=0.003). None of the biomarkers showed an efficient diagnostic performance in distinguishing between PPC and CP (Fig. 1G).

A combination of any two or three of these biomarkers improved the diagnostic performance in distinguishing between PC and CP. Combination of the three biomarkers showed the largest AUC (0.982; $\mathrm{P}<0.001)$. Combination of any two of the three biomarkers showed that MIC-1 combined with ULBP2 produced the optimal diagnostic performance (AUC, 0.977; P<0.001; Fig. 2).

Correlations between ULBP2, MIC-1 and PC survival. Survival data were analyzed using the Kaplan-Meier method with log-rank test for comparison. In this study, the median was set as the cut-off point. It was found that MIC-1 was correlated with the prognosis of PC. A higher serum level of MIC-1 indicated a worse prognosis. The median survival of PC patients with a serum MIC-1 level of $\leq 1,932 \mathrm{pg} / \mathrm{ml}$ was 
Table IV. AUC of different biomarkers in distinguishing stage1-2 PC or stage 3-4 PC from CP or NC.

\begin{tabular}{|c|c|c|c|c|}
\hline PC stage & CPAUC & $95 \% \mathrm{CI}$ & NC AUC & $95 \% \mathrm{CI}$ \\
\hline \multicolumn{5}{|c|}{ Stage 1-2 PC } \\
\hline MIC-1 & 0.831 & $0.754-0.908$ & 0.964 & 0.929-0.999 \\
\hline ULBP2 & 0.925 & $0.873-0.978$ & 0.896 & $0.831-0.961$ \\
\hline CA19-9 & 0.802 & $0.714-0.891$ & 0.892 & $0.826-0.959$ \\
\hline \multicolumn{5}{|c|}{ Stage 3-4 PC } \\
\hline MIC-1 & 0.809 & $0.730-0.889$ & 0.954 & 0.916-0.992 \\
\hline ULBP2 & 0.913 & $0.853-0.972$ & 0.875 & $0.800-0.950$ \\
\hline CA19-9 & 0.795 & $0.713-0.877$ & 0.888 & $0.822-0.955$ \\
\hline
\end{tabular}

PC, pancreatic cancer; CP, chronic pancreatitis; PPC, pre-pancreatic cancer; NC, normal controls; CA19-9, carbohydrate antigen 19-9; ULBP2, UL16-binding protein 2; MIC-1, macrophage inhibitory cytokine-1; AUC, area under the curve; CI, confidence interval.
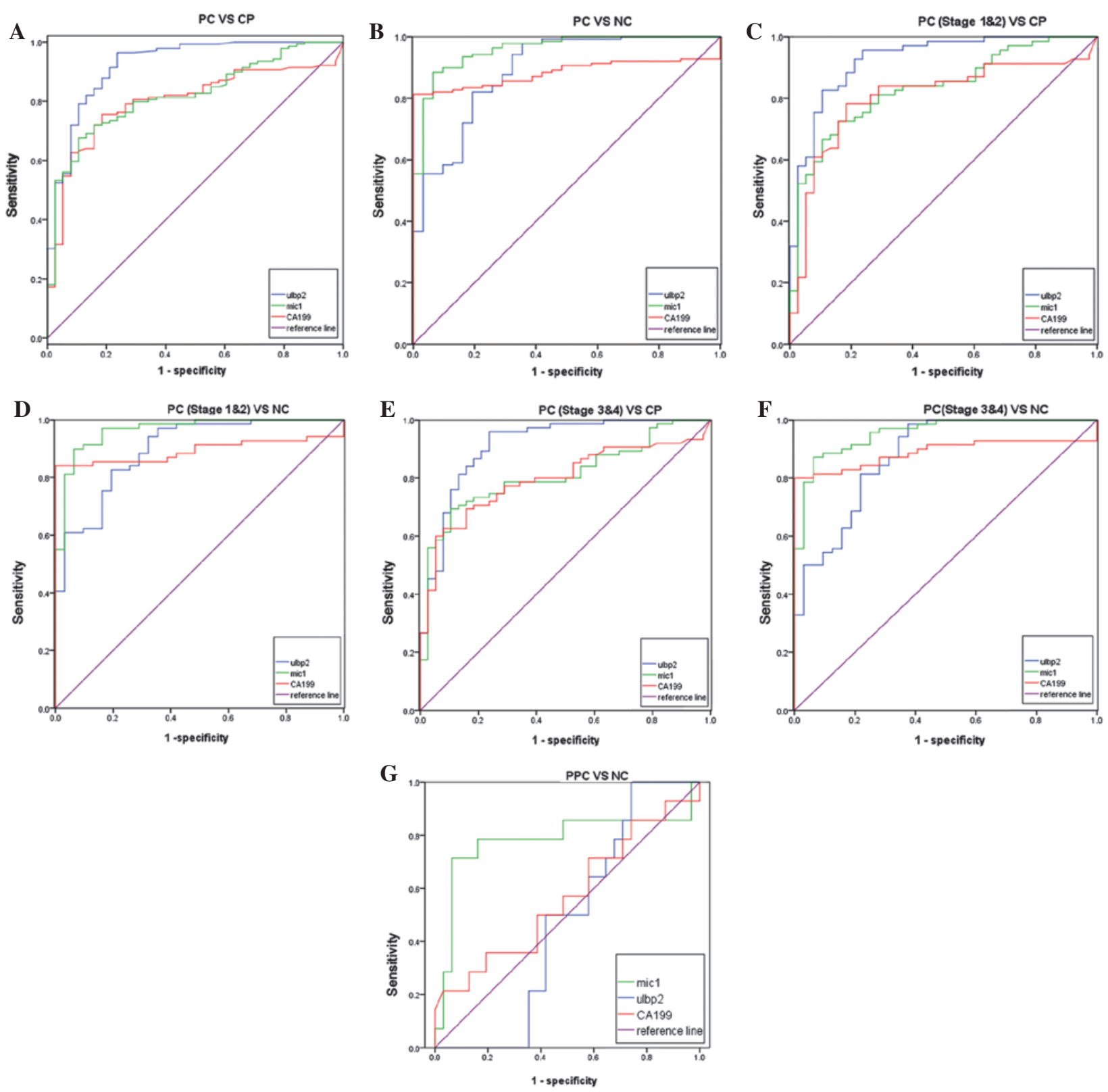

Figure 1. Diagnostic performance of ULBP2, MIC-1 and CA19-9 alone. (A) PC vs. CP. (B) PC vs. NC. (C) Stage 1-2 PC vs. CP. (D) Stage 1-2 PC vs. NC. (E) Stage 3-4 PC vs. CP. (F) Sstage 3-4 PC vs. NC. P-values of all receiver operating characteristic curves were $<0.001$. (G) PPC vs. NC; only serum MIC-1 could efficiently distinguish between PPC and NC (AUC, 0.781; 95\% CI, 0.599-0.963; P=0.003). PC, pancreatic cancer; CP, chronic pancreatitis; PPC, pre-pancreatic cancer; NC, normal controls; CA19-9, carbohydrate antigen 19-9; ULBP2, UL16-binding protein 2; MIC-1, macrophage inhibitory cytokine-1; AUC, area under the curve. 

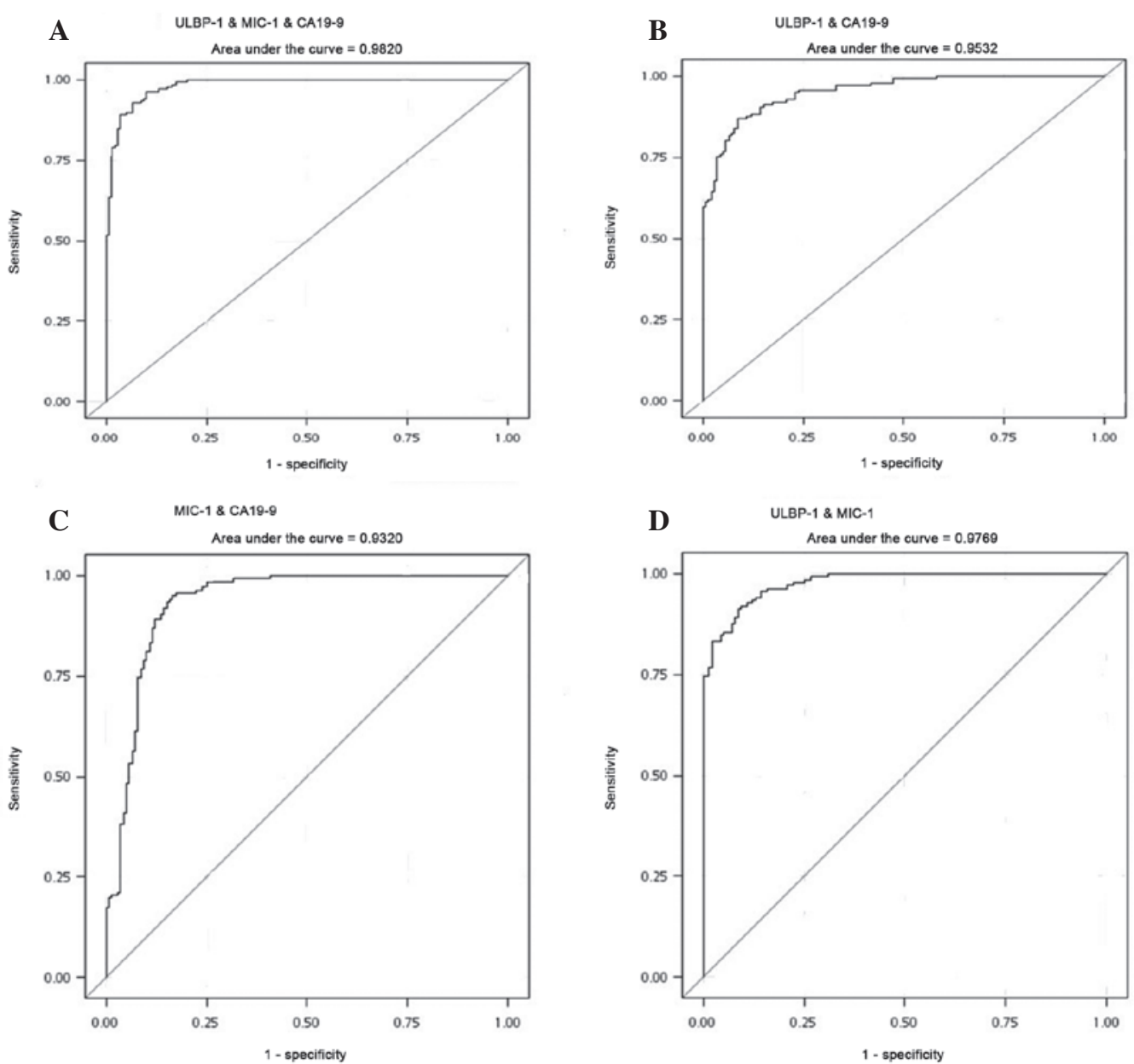

Figure 2. Combination of two or three markers for pancreatic cancer diagnosis compared with CA19-9 alone. (A) Combination of ULBP2, MIC-1 and CA19-9; AUC, 0.982. (B) Combination of ULBP2 and CA19-9; AUC, 0.953. (C) Combination of MIC-1 and CA19-9; AUC, 0.932. (D) Combination of ULBP2 and MIC-1; AUC, 0.977. P-values of all receiver operating characteristic curves were <0.001. CA19-9, carbohydrate antigen 19-9; ULBP2, UL16-binding protein 2; MIC-1, macrophage inhibitory cytokine-1; AUC, area under the curve.

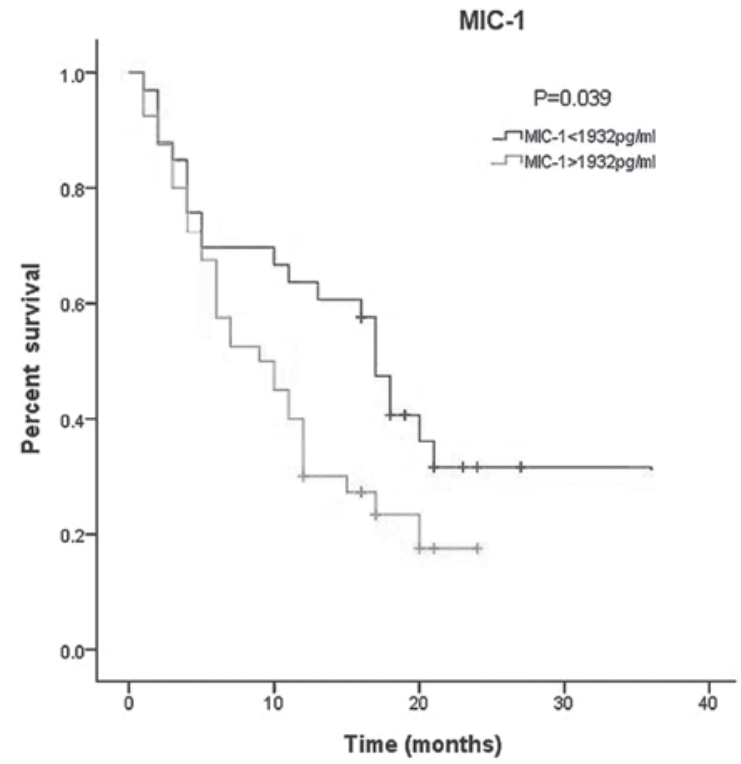

Figure 3. Overall survival of PC stratified by serum MIC-1. Median survival time was $18.66 \pm 2.43$ months in PC patients with a serum MIC-1 level of $\leq 1,932 \mathrm{pg} / \mathrm{ml}$ (95\% CI, 13.91-23.42) vs. 15.617 \pm 2.44 months in patients with a serum MIC-1 level of $>1,932 \mathrm{pg} / \mathrm{ml}$ (95\% CI, 10.83-20.41). $\mathrm{P}=0.039$ and $\chi^{2}=4.239$. PC, pancreatic cancer; MIC-1, macrophage inhibitory cytokine-1; $\mathrm{CI}$, confidence interval.
$18.66 \pm 2.43$ months (95\% CI, 13.91-23.42), while a higher MIC-1 level was associated with a shorter survival duration averaging 15.617 \pm 2.44 months (95\% CI, 10.83-20.41) (Fig. 3). No correlation was found between the serum ULBP2 level and PC prognosis.

\section{Discussion}

CA19-9 is currently the most commonly used serological marker in the diagnosis of PC, but its performance in early diagnosis is limited. Therefore, it is more often used in the follow-up of PC patients after surgery or chemotherapy (10-12). In addition, elevated serum CA19-9 levels have been reported in a number of gastrointestinal diseases, such as pancreatitis, hepatitis and biliary obstruction. Serum CA19-9 levels are particularly increased in patients with biliary obstruction (13). These findings compromise the specificity of CA19-9. Clearly, determining novel $\mathrm{PC}$ biomarkers to circumvent this drawback of CA19-9 has good prospects for development. In the present study, it was found that serum MIC-1 and ULBP2 improved the diagnostic accuracy in differentiating between PC, CP and NC, and that serum MIC-1 levels could be used to predict survival in PC patients. 
ULBP2 and MIC-1 have been reported to be overexpressed in various types of cancer tissue and to be secreted by cancer cells, including PC cells (14-17). This is consistent with the findings of the present study showing that serum ULBP2 and MIC-1 levels were significantly higher in the PC patients than in the $\mathrm{NC}$ and $\mathrm{CP}$ patients. In addition, the study was the first to find that serum MIC-1 and ULBP2 expression was significantly elevated in the PPC patients compared with the $\mathrm{NC}(\mathrm{P}=0.003)$, while there was no significant difference in CA19-9 between the two groups, suggesting that ULBP2 and MIC-1 could be used for the early diagnosis of PC.

The early diagnosis of PC remains a clinical challenge at present. Although numerous studies have demonstrated that multiple serum biomarkers, including peptidylglycine $\alpha$-amidating monooxygenase 4 , heat shock protein 27 and tumor-specific growth factor, are elevated in PC, the diagnostic performance of these biomarkers alone is not sufficient for clinical application, particularly due to their limited capacity for the early diagnosis of PC (18). The present study first confirmed that ULBP2 and MIC-1 alone outperformed CA19-9 in identifying PC, with high specificity and sensitivity. The combination of ULBP2, MIC-1 and CA19-9 enhanced the efficacy of the PC diagnosis. Subsequently, it was found that ULBP2 and MIC-1 performed better than CA19-9 in distinguishing early-stage, stage 1-2, PC. More significantly, by plotting ROC curves and calculating the AUC, it was found that only MIC-1 had the diagnostic capability for distinguishing between PPC and NC (AUC, 0.781; 95\% CI, 0.599-0.963; $\mathrm{P}=0.003)$. Therefore, serum ULBP2 and MIC-1 are useful biomarkers for the diagnosis of PC, particularly in the diagnosis of early-stage PC.

In addition, this study also analyzed the correlation between ULBP2 and MIC-1, and PC prognosis. The result showed that serum MIC-1 was correlated with PC prognosis.

The molecular mechanism of ULBP2 and MIC-1 underlying the development of PC remains unclear. ULBP2 is one of the ligands for NKG2D. When the normal epithelium is transformed, the stress-induced ligands for NKG2D (human MIC and ULBP antigens) may be expressed on the cell surface (19). ULBP2 on the tumor cell surface can bind to NKG2D receptors on immune cells, such as $\mathrm{NK}$ cells and $\mathrm{CD}^{+} \mathrm{T}$ cells, ultimately inducing the innate immune response of killing and scavenging tumor cells (20). ULBP2 expression is regulated by tumor suppressors at the transcriptional and post-translational levels. For example, p53 functions as a direct transcriptional activator of ULBP2 and represses ULBP2 translation by upregulating microRNA (miR)-34a and miR-34b/c. The ultimate ULBP2 level is determined by the balance of the two regulatory mechanisms (21).

MIC-1 was first reported by Bootcov et al in 1997, and is recognized as a divergent member of the transforming growth factor- $\beta$ (TGF- $\beta$ ) superfamily, which plays a complex role in several human diseases, including cancer (7). MIC-1 can serve as a potential diagnostic and prognostic biomarker for certain cancers (22). A number of studies have shown that MIC-1 can activate multiple signaling pathways, including the focal adhesion kinase signaling pathway, the extracellular signal regulated kinase $1 / 2$ signaling pathway and the phosphatidylinositol 3'-kinase-Akt intracellular pathway, although the identity of the MIC-1 binding ligand is not clear (23-25). MIC-1 is the only known cytokine to regulate secreted p53, which is expressed strongly in the presence of active p53 $(26,27)$. Although MIC-1 plays an antitumor role in the early stages of cancer, it promotes invasion and metastasis in advanced stages. Johnen et al reported that serum MIC-1 was closely associated with sustained weight loss in cancer patients (28).

In conclusion, the present study demonstrated that ULBP2 and MIC-1 could be used potentially as serum biomarkers in the diagnosis of $\mathrm{PC}$, particularly when they are used in combination. In addition, serum MIC-1 could be used to predict the PC outcome. Further studies are required to clarify the molecular mechanisms of ULBP2 and MIC-1 underlying the development of PC.

\section{References}

1. Berrino F, De Angelis R, Sant M, et al; EUROCARE Working Group: Survival for eight major cancers and all cancers combined for European adults diagnosed in 1995-99: results of the EUROCARE-4 study. Lancet Oncol 8: 773-783, 2007.

2. Siegel R, Naishadham D and Jemal A: Cancer statistics, 2012. CA Cancer J Clin 62: 10-29, 2012.

3. Ishikawa O, Ohigashi H, Imaoka S, et al: Minute carcinoma of the pancreas measuring $1 \mathrm{~cm}$ or less in diameter - collective review of Japanese case reports. Hepatogastroenterology 46: 8-15, 1999.

4. Yachida S, Jones S, Bozic I, et al: Distant metastasis occurs late during the genetic evolution of pancreatic cancer. Nature 467: 1114-1117, 2010.

5. Pannala R, Basu A, Petersen GM and Chari ST: New-onset diabetes: a potential clue to the early diagnosis of pancreatic cancer. Lancet Oncol 10: 88-95, 2009.

6. Cosman D, Müllberg J, Sutherland CL, et al: ULBPs, novel MHC class I-related molecules, bind to CMV glycoprotein UL16 and stimulate NK cytotoxicity through the NKG2D receptor. Immunity 14: 123-133, 2001.

7. Bootcov MR, Bauskin AR, Valenzuela SM, et al: MIC-1, a novel macrophage inhibitory cytokine, is a divergent member of the TGF-beta superfamily. Proc Natl Acad Sci USA 94: 11514-11519, 1997.

8. Hruban RH, Maitra A, Kern SE and Goggins M: Precursors to pancreatic cancer. Gastroenterol Clin North Am 36: 831-849, 2007.

9. AJCC Cancer Staging Manual. 6th edition. Springer-Verlag, New York, NY, 2002.

10. Gui JC, Yan WL and Liu XD: CA19-9 and CA242 as tumor markers for the diagnosis of pancreatic cancer: a meta-analysis. Clin Exp Med 14: 225-233, 2014.

11. Wu E, Zhou S, Bhat K and Ma Q: CA 19-9 and pancreatic cancer. Clin Adv Hematol Oncol 11: 53-55, 2013.

12. Hartwig W, Strobel O, Hinz U, et al: CA19-9 in potentially resectable pancreatic cancer: perspective to adjust surgical and perioperative therapy. Ann Surg Oncol 20: 2188-2196, 2013.

13. Singh S, Tang SJ, Sreenarasimhaiah J, et al: The clinical utility and limitations of serum carbohydrate antigen (CA19-9) as a diagnostic tool for pancreatic cancer and cholangiocarcinoma. Dig Dis Sci 56: 2491-2496, 2011.

14. Chang YT, Wu CC, Shyr YM, et al: Secretome-based identification of ULBP2 as a novel serum marker for pancreatic cancer detection. PLoS One 6: e20029, 2011.

15. Koopmann J, Buckhaults P, Brown DA, et al: Serum macrophage inhibitory cytokine 1 as a marker of pancreatic and other periampullary cancers. Clin Cancer Res 10: 2386-2392, 2004.

16. Koopmann J, Rosenzweig CN, Zhang Z, et al: Serum markers in patients with resectable pancreatic adenocarcinoma: macrophage inhibitory cytokine 1 versus CA19-9. Clin Cancer Res 12: 442-446, 2006.

17. Yamaguchi K, Chikumi H, Shimizu A, et al: Diagnostic and prognostic impact of serum-soluble UL16-binding protein 2 in lung cancer patients. Cancer Sci 103: 1405-1413, 2012.

18. Bünger S, Laubert T, Roblick UJ and Habermann JK: Serum biomarkers for improved diagnostic of pancreatic cancer: a current overview. J Cancer Res Clin Oncol 137: 375-389, 2011.

19. Cerwenka A and Lanier LL: Natural killer cells, viruses and cancer. Nat Rev Immunol 1: 41-49, 2001.

20. Champsaur M and Lanier LL: Effect of NKG2D ligand expression on host immune responses. Immunol Rev 235: 267-285, 2010. 
21. Li H, Lakshmikanth T, Garofalo C, et al: Pharmacological activation of $\mathrm{p} 53$ triggers anticancer innate immune response through induction of ULBP2. Cell Cycle 10: 3346-3358, 2011.

22. Brown DA, Hance KW, Rogers CJ, et al: Serum macrophage inhibitory cytokine-1 (MIC-1/GDF15): a potential screening tool for the prevention of colon cancer? Cancer Epidemiol Biomarkers Prev 21: 337-346, 2012.

23. Senapati S, Rachagani S, Chaudhary K, et al: Overexpression of macrophage inhibitory cytokine-1 induces metastasis of human prostate cancer cells through the FAK-RhoA signaling pathway. Oncogene 29: 1293-1302, 2010.

24. Chen SJ, Karan D, Johansson SL, et al: Prostate-derived factor as a paracrine and autocrine factor for the proliferation of androgen receptor-positive human prostate cancer cells. Prostate 67: 557-571, 2007.
25. Proutski I, Stevenson L, Allen WL, et al: Prostate-derived factor - a novel inhibitor of drug-induced cell death in colon cancer cells. Mol Cancer Ther 8: 2566-2574, 2009.

26. Tan M, Wang Y, Guan K and Sun Y: PTGF-beta, a type beta transforming growth factor (TGF-beta) superfamily member, is a p53 target gene that inhibits tumor cell growth via TGF-beta signaling pathway. Proc Natl Acad Sci USA 97: 109-114, 2000

27. Li PX, Wong J, Ayed A, et al: Placental transforming growth factor-beta is a downstream mediator of the growth arrest and apoptotic response of tumor cells to DNA damage and p53 overexpression. J Biol Chem 275: 20127-20135, 2000.

28. Johnen H, Lin S, Kuffner T, et al: Tumor-induced anorexia and weight loss are mediated by the TGF-beta superfamily cytokine MIC-1. Nat Med 13: 1333-1340, 2007. 